\title{
Planar Antenna-Coupled Transition-Edge Hot Electron Microbolometer
}

\author{
Shafinaz Ali, Lance D. Cooley, Dan McCammon, Kari L. Nelms, John Peck, Daniel Prober, Daniel Swetz, \\ Peter T. Timbie, and Daniel van der Weide
}

\begin{abstract}
We describe a new type of bolometric detector for millimeter and submillimeter wavelengths. The detector is a variant of the Transition Edge Sensor (TES), which has recently been used to build bolometers. In this version of the TES, we couple radiation from a planar antenna to an absorbing normal metal film which is electrically connected to a superconducting thin film. The lateral dimensions of the absorber and TES are 10 microns. At low temperatures, the thermal isolation between the electrons and the lattice in the absorber and the superconductor allows the electrons to heat up. We call this device a Transition-edge Hot-electron Microbolometer (THM). These detectors could have numerous advantages for low-background measurements in the far-IR, such as, background-limited sensitivity, short time constant, wide spectral range, immunity to cosmic rays, low microphonic noise and simple readout electronics. We are currently building a low-frequency scale model of the planar antenna to characterize microwave properties of the system.
\end{abstract}

Index Terms-Bolometers, hot-electron, superconducting sensors.

\section{INTRODUCTION}

$\mathbf{T}$ HE millimeter and the submillimeter wavelengths of the electromagnetic spectrum hold a wealth of information about the evolution of the universe. In particular, precise measurements of the power spectrum of the temperature and polarization variations of the Cosmic Microwave Background (CMB) radiation can answer basic questions in cosmology [1]. The signal from the $2.7 \mathrm{~K} \mathrm{CMB}$ is exceedingly small; the temperature fluctuations (anisotropy) in the CMB are 1 part in 100000 and the predicted polarized signal from the radiation is at least a factor of 10 times smaller. Highly sensitive bolometers are the detectors of choice in future CMB experiments. These detectors are reaching the background-limited noise limit for arrays of a few to $\sim 100$ detectors. One way of increasing sensitivity is to build a larger array of detectors. With conventional bolometers these arrays are limited in size due to fabrication difficulties. The transition edge sensors can be produced by standard lithographic techniques, so arrays of

Manuscript received August 6, 2002. This work was supported by NASA under Grant NAG5-10258.

S. Ali, D. McCammon, K. L. Nelms, D. Swetz, and P. T. Timbie are with the Department of Physics, University of Wisconsin-Madison, Madison, WI 53706 USA (e-mail: ali@wisp.physics.wisc.edu).

L. D. Cooley is with the Department of Materials Science and Engineering and the Applied Superconductivity Center, University of Wisconsin-Madison, Madison, WI 53706 USA.

J. Peck and D. van der Weide are with the Department of Electrical and Computer Engineering, University of Wisconsin-Madison, Madison WI 53706 USA.

D. Prober is with the Department of Applied Physics, Yale University, New Haven, CT 06520 USA.

Digital Object Identifier 10.1109/TASC.2003.813676
100-1000 elements seem feasible. Detectors that are matched across the array are highly desirable.

One approach for building such large arrays is to use bolometers based on superconducting transition edge sensors (TES). A TES involves a superconducting thin-film biased at the middle of its superconducting-normal transition curve. The sensor is maintained at the middle of the transition by electrothermal feedback [2]. The absorbed power is proportional to the feedback current. The optimization of the first superconducting bolometer was described by Clarke et al. [3]. Since then this kind of sensor has been successfully used for $\mathrm{X}$-ray detectors [4], and for infrared bolometers [5]. A major advantage of TES bolometers and microcalorimeters is that they can be read out by SQUID amplifiers, which can be used in a variety of multiplexing schemes. In these TES devices the absorber is electrically separate from the superconducting film itself. Incident energy is captured by an absorber which then heats the phonons in the substrate of the bolometer. The quasiparticles in the superconducting film are heated in turn by these phonons.

In a hot-electron bolometer the incident radiation heats the electron system in the thermally-sensitive element directly. Mees et al. [6], proposed a scheme where radiation is coupled via a planar antenna to a thin normal metal film which terminates the antenna and absorbs the power. Antenna coupling to radiation allows the thermally active region to be much smaller than a wavelength. Furthermore, planar antennas are compatible with planar superconducting transmission lines which can be used for constructing microwave filters, multiplexers, etc.

In the hot-electron microbolometer proposed by Nahum et al. [7], the weak coupling between the electrons and the phonons in metals at low temperature produces a large temperature rise of the electrons for a small rise in the input power. The temperature of the electrons heated in this film is determined by measuring the tunnelling current through a NIS tunnel junction. An enormous advantage of this approach is that the thermal isolation of the bolometer does not require micromachining or assembly of thin structures. The scheme was demonstrated by Nahum and Martinis [8]. Similar hot-electron approaches have been suggested in which a TES simultaneously acts as absorber and detector. In this case the incident power is absorbed directly by the quasiparticles in the superconducting film. Cabrera et al. [9], have demonstrated a microcalorimeter of this type where optical and UV photons are absorbed directly from free space into a superconducting film of tungsten. Karasik et al. [10], have proposed to use the TES as the termination of a planar antenna; they call this a hot-electron direct detector (HEDD). Our plan 
is similar to that of Karasik et al., except we have decided to separate the functions of the absorber and the TES. We call this device a transition-edge hot-electron microbolometer (THM).

\section{DESIGN}

\section{A. Transition-Edge Hot-Electron Microbolometer (THM)}

In this scheme a planar antenna is terminated in a thin normal metal film. The film is coupled directly to the quasiparticle system of a TES. The goal is for the hot electrons in the absorber to heat the quasiparticles in the TES. The purpose of forming a separate absorber and TES is to allow flexibility in the optimization of the two components. In particular, the absorbing film's impedance can be chosen to match the antenna or transmission line $(10 \Omega-50 \Omega)$ while the TES impedance can be chosen to match to the readout SQUID amplifier (optimum is usually $<1 \Omega$ ).

\section{B. Optimization of $G$ and $T_{\mathrm{Bath}}$}

Here we describe how the design of the THM can be optimized for a particular application: measurements of the $\mathrm{CMB}$ from a cooled telescope in space. We estimate the sensitivity and time constant for this case because it is both relevant and simple to discuss. The optimization is meant as an example; there are many potential uses for these devices, particularly in low-background environments.

For any bolometer, the sensitivity is determined by a variety of sources of noise. These include fluctuations in the photon stream absorbed on the detector ("photon noise"), thermal fluctuations ("phonon noise") in the thermal link between the bolometer and its heat bath, Johnson noise in the thermistor, and amplifier noise. Bolometer noise is expressed in terms of noise equivalent power (NEP), which has units of $W / \sqrt{\mathrm{Hz}}$. The various noise contributions are added in quadrature. For simplicity we consider only the first two noise sources and assume that the others can be made negligible in a proper bolometer design. The time constant depends on the ratio of the bolometer's heat capacity $C$ to the thermal conductivity $G$ between bolometer and heat bath: $\tau=C / G$; electro-thermal feedback reduces the time constant even further [2], [5].

Photon noise arises from fluctuations in the arrival rate of the photons absorbed by the detector. In our example, it is the fluctuations in the arrival of the signal photons themselves that set a fundamental limit to the detector's sensitivity. Assume that the bolometer is illuminated only by the CMB (blackbody with $T_{\mathrm{CMB}}=2.726 \mathrm{~K}$ and emissivity $\epsilon=1$ ) and that the bolometer absorbs a single mode and single polarization of this radiation with $50 \%$ efficiency $(\eta=0.5)$ in a $30 \%$ bandwidth centered at a frequency of $90 \mathrm{GHz}$. The expression for the photon noise absorbed in the detector in this case can be expressed as in the following equation [11]:

$$
\mathrm{NEP}_{\text {Photon }}^{2}=\frac{2 \epsilon \eta(h v)^{2} \Delta v}{\left(e^{h v / k_{B} T_{\mathrm{CMB}}}-1\right)}\left(1+\frac{\epsilon \eta}{\left(e^{h v / k_{B} T_{\mathrm{CMB}}}-1\right)}\right) .
$$

With the parameters given above, this expression yields

$$
\mathrm{NEP}_{\text {Photon }}=5.2 \times 10^{-18} \mathrm{~W} / \sqrt{\mathrm{Hz}} \text {. }
$$

Phonon noise is given by $\mathrm{NEP}_{\text {Phonon }}{ }^{2}=4 k_{B} T_{\text {Bolo }}^{2} G$, where $k_{B}$ is Boltzmann's constant, $T_{\text {Bolo }}$ is the temperature of the bolometer itself (which is hotter than the bath temperature), and $G$ is the thermal conductivity between the bolometer and the heat bath and has units of $W / K$. For a fixed bath temperature $T_{\text {Bath }}$ and absorbed optical power $P$, there is an optimum value of $G$; if $G$ is too high, phonon noise increases, while if $G$ is too low, the bolometer gets too hot and again the phonon noise increases. In the case of the hot-electron microbolometer, $T_{\mathrm{Bolo}}$ is equal to the temperature of the electrons, $T_{e}$, and $T_{\text {Bath }}$ equals the temperature of the phonons $T_{p h}$ and the lattice.

Here we determine the optimum value of $G$ and $T_{\text {Bath }}$ for our idealized case. The absorbed power in the Rayleigh-Jeans approximation is $P=\eta k_{B} T_{\mathrm{CMB}} \Delta \nu$. This power will heat the bolometer above the bath temperature to $T_{\text {Bolo }}=T_{\text {Bath }}+$ $P / G$. (In this simplified calculation we are neglecting the electrical power dissipated by the bias current in the TES). After inserting this expression for $T_{\text {Bolo }}$ into the above equation for $\mathrm{NEP}_{\text {Phonon, }}$, one can show that the optimum value of $G$ is $G=$ $P / T_{\text {Bath }}$ and that the optimum operating temperature of the bolometer is $T_{\text {Bolo }}=2 T_{\text {Bath }}$.

The phonon noise decreases as $T_{\mathrm{Bath}}$ is reduced. We can determine the maximum acceptable value of $T_{\mathrm{Bath}}$ as follows. If we design the bolometer so that $\mathrm{NEP}_{\text {Phonon }}$ is equal to $(1 / 2) \mathrm{NEP}_{\text {Photon }}$ then the combined noise will only be $11 \%$ above the background photon noise limit. That is, $\mathrm{NEP}_{\text {Phonon }}{ }^{2}=4 k_{B}\left(2 T_{\text {Bath }}\right)^{2}\left(P / T_{\text {Bath }}\right) \leq$ (1/4) $\mathrm{NEP}_{\text {Photon }}{ }^{2}$. This gives $T_{\text {Bath }} \leq 0.054 \mathrm{~K}$. Assuming $T_{\text {Bath }}=0.050 \mathrm{~K}$, the optimum value of $G$ is $1.2 \times 10^{-11} \mathrm{~W} / \mathrm{K}$.

\section{Thermal Conductivity to Heat Bath}

Here we explore how to use the thermal decoupling between the electrons and the phonons in a normal metal to provide the optimum thermal conductivity $G$ determined in Section II-B. In a normal metal at low temperatures the power conducted from the electron system to the phonon system is $P_{e-p h}=\Sigma V\left(T_{e}^{5}-T_{p h}{ }^{5}\right)$, where $\Sigma$ is a constant that depends on the material, $V$ is the volume of the metal, and $T_{e}$ and $T_{p h}$ are the temperatures of the electrons and the phonons respectively [12]. The corresponding thermal conductivity is given by $G_{e-p h}=d P_{e-p h} / d T=5 \Sigma V T^{4}$. We use this expression to determine the conductivity from the electrons to the phonons in the normal metal absorbing film. We assume that the quasiparticle-phonon coupling in the TES film obeys the same expression because the TES is biased in the the middle of the superconducting-normal transition [13]. The TES film is electrically (and hence, thermally) connected to the absorber. In $\mathrm{Cu}$ films at $T_{p h}=0.040 \mathrm{~K}$, Nahum and Martinis measured $\Sigma=3.7 \mathrm{nWK}^{-5} \mu \mathrm{m}^{-3}$ [8]. For our system, with $T_{p h}=T_{\text {Bath }}=0.050 \mathrm{~K}, T_{e}=2 T_{\text {Bath }}$, and $\Sigma$ as above, we obtain $G_{e-p h}=1.2 \times 10^{-11} \mathrm{~W} / \mathrm{K}$ when $V=6.2 \mu \mathrm{m}^{3}$. The volume of the TES is chosen to be smaller than that of the absorber so that the electron-phonon coupling in the absorber provides the dominant thermal path to the lattice.

There are two other thermal paths between the electrons and the lattice. These are from the absorber to the antenna (or superconducting transmission line) and from the TES itself to the superconducting leads that read out the TES. In the former case, 
the electrons are prevented from entering the superconductor by its energy gap (Andreev reflection). A similar effect occurs in the second case, where the superconducting energy gap in the TES will be lower than that of the superconducting leads.

\section{Internal Thermal Conductance \& Impedance Matching}

Now that the operating temperature and the volume of the absorber and TES have been determined, we need to determine the dimensions of these components. There are two constraints: first, the absorbing film must have an impedance that matches that of the planar antenna or transmission line to which it is connected and second, the thermal conductance within the device must be greater than the thermal conductance to the heat bath, which is dominated by $G_{e-p h}$.

These two constraints are related by the Wiedemann-Franz law in the following manner [14]. $G_{W-F}=L_{0} T_{e} / R$, where $G_{W-F}$ is the thermal conductance from one end of the film to other, $T_{e}$ is the temperature of the electrons in the film, $R$ is the electrical resistance from one end of the film to the other, and $L_{0}=(1 / 3)\left(\pi k_{B} / e\right)^{2}=2.44 \times 10^{-8} \mathrm{~W} \Omega / \mathrm{K}^{2}$ is the Lorenz number. Because the resistance of the film needs to be in the range of 10-50 $\Omega$ to match to the RF circuit, we can immediately determine that $G_{W-F}=2.44 \times 10^{-10}$ to $4.88 \times 10^{-11} \mathrm{~W} / \mathrm{K}$. This value is a factor of 5 to 25 greater than the electron-phonon conductance so that we can treat the electron bath approximately as isothermal.

For a film of length $L$, width $w$, thickness $t$, and electrical conductivity $\sigma, R=(1 / \sigma)(L / w t)$. The electrical conductivity can be written as $\sigma=\left(e / \pi k_{B}\right)^{2} \gamma \lambda_{e} v_{F}$ [14] where $\lambda_{e}$ is the mean free path of the electrons, $\gamma$ is the Sommerfeld constant (in units of $\left.\left[\mathrm{J} / \mathrm{m}^{3} \mathrm{~K}^{2}\right]\right)$, and $v_{F}$ is the Fermi velocity of the electrons. The Fermi velocity [14] is $v_{F}=\left(\hbar / m_{e}\right)\left(3 \pi^{2} N_{0} / V_{m}\right)^{1 / 3}$, where $N_{0}$ is Avogadro's number and $V_{m}$ is the volume of one mole of the metal. $v_{F}$ is approximately $10^{6} \mathrm{~m} / \mathrm{s}$ for copper. Assuming that the mean free path of the electrons is determined by the film thickness, so that $t=\lambda_{e}$ (which will be of the order of $20 \mathrm{~nm}$ ), the film electrical resistance can be rewritten as $R=3 L_{0} L / \lambda_{e}^{2} v_{F} \gamma w$. For copper, $\gamma=97 \mathrm{~J} / \mathrm{m}^{3} \mathrm{~K}^{2}$ [14] and the resistance becomes $R=7.5 \times 10^{-16} \mathrm{~L} / \mathrm{wt}^{2} \Omega$. As shown above, the optimum value of $G_{e-p h}$ occurs when the volume of the absorber is $V=6.2 \mu \mathrm{m}^{3}$. For an absorber with resistance $R=10 \Omega$ this requirement can be met by choosing the thickness to be $t=23 \mathrm{~nm}$ and $L \times w=44 \mu \mathrm{m} \times 6 \mu \mathrm{m}$.

\section{E. Thermal Time Constant}

Finally, we want the time constant of the detectors to be short enough to permit a variety of scan strategies for observing the sky. $\tau=C_{e} / G_{e-p h}$ where $C_{e}=\gamma T_{e} V$ is the heat capacity of the electron system [14] and $G_{e-p h}$ is given above. We obtain $\tau=\gamma T_{e} V / 5 \Sigma V T_{e}^{4}$. Again for the case of copper, we obtain $\tau \sim 5 \mu \mathrm{s}$. Note that the time constant is independent of volume. This time constant is more than adequate for most applications. Electrothermal feedback will shorten it still further [2], [5].

\section{F. Planar Antenna}

A wide variety of planar antennas make good candidates for coupling to millimeter and submillimeter wavelength detectors. THM's can be easily coupled to planar antennas via low-loss superconducting microstrip transmission lines. Planar double slot antennas are particularly useful because they produce nearly Gaussian beams. A pair of dual slot antennas oriented at $90^{\circ}$ to each other will couple to two orthogonal polarizations which makes them a perfect choice for polarization measurements. Chattopadhyay and Zmuidzinas [15], [16] have used a dual-polarization arrangement of slot antennas coupled to coherent detectors. Several research groups [e.g., [17], [18]] are also developing designs in the submillimeter wavelength region to couple TES devices to polarization-sensitive planar antennas. We plan to fabricate the slot antenna on a Si wafer coated with thin layers of superconducting $\mathrm{Nb}$. The groundplane and the microstrip transmission lines are separated by a layer of insulator, such as $\mathrm{SiO}$. The beam width of the planar antenna can be adjusted by placing the antenna on a thick hyperhemispherical silicon lens [19]. The lens maintains the gaussicity and the directivity of the beam pattern and suppresses any substrate modes.

\section{MEASUREMENTS}

\section{A. Simulation and Scale Model}

A simulation of the planar antenna coupled to a microstrip transmission line was performed with the commercially available ADS software (version 2, Agilent Technologies, Palo Alto, CA). The substrate material separating the metal microstrip and slot layers is set to have $\epsilon_{r}=6$ to approximate $\mathrm{SiO}$. A typical thickness for this layer in a fabricated antenna would be about 1 $\mu \mathrm{m}$ - a length very small compared to the length of the slot and feed line. To facilitate scale modeling with reasonable model dimensions, two simulations were performed: one with a $1 \mu \mathrm{m}$ substrate and one with a thicker, $21 \mu \mathrm{m}$, substrate to allow the scale model to be patterned on $0.25 \mathrm{~mm}$ thick Duroid (Rogers Inc.) material. The additional substrate thickness does not significantly affect the position of the second slot resonance.

The slot length was chosen to place the second, or full-wave, resonance near $100 \mathrm{GHz}$. The second resonance was chosen over the relatively high-impedance first resonance to enable broad-band matches to low-impedance transition edge detectors. The slot width was chosen to be the same as the microstrip feed, which was chosen to be the minimum reliable line width possible with the scale model fabrication. The overlap of the microstrip feed was chosen to be $\lambda / 4$ at $100 \mathrm{GHz}$, where $\lambda$ is the wavelength within the microstrip structure. The feedline extends $\lambda / 2$ on the other side of the slot to enable simulation of this intermediate structure with the feed point positioned away from the near field of the antenna. After these simulations were done, the results were used to design a quarter-wave transformer to raise the input impedance of the system for testing with $50 \Omega$ equipment. This matching network does not significantly shift the position of the simulated resonance. The final structure separates the transformer from the feed point by a length of $50 \Omega$ line. The scale model was made to resonate at around $8.5 \mathrm{GHz}$. 


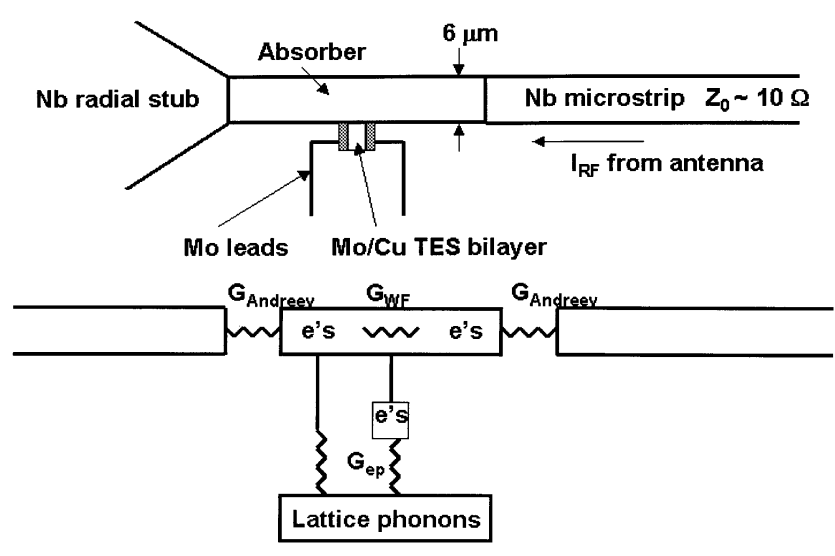

Fig. 1. Top figure shows a resistive normal metal film acting as a termination of a microstrip transmission line with an impedance of $10 \Omega$. The film overlaps an adjacent TES sensor and forms electrical and thermal contact with it. Bottom figure is a schematic of the thermal circuit for the THM. In order to treat the electrons as isothermal, we require the internal thermal conductivity of the absorber (and TES) to be much greater than the electron-phonon conductivity. That is, $G_{W-F} \gg G_{e-p h}$. In addition, $G_{e-p h}$ must be chosen so that the thermal fluctuations in electron-phonon thermal link are small compared to the photon noise from the observed source.

\section{FABRICATION}

We have fabricated TES devices at the Wisconsin Center for Applied Microelectronics (WCAM) by depositing by e-beam evaporation a bilayer of Mo and $\mathrm{Cu}$ on $\mathrm{Si}$ wafers. Transition temperature of a bilayer is dependent on the thickness ratio of the superconducting to the normal metal [20]; a TES device with $40 \mathrm{~nm} / 174 \mathrm{~nm}$ of Mo/Cu layers is measured to have a $T_{c}$ of 350 $\mathrm{mK}$. The $\mathrm{Mo} / \mathrm{Cu}$ bilayer is patterned by wet etching and dry plasma etching. In order to form transmission lines and antennas we have deposited $300 \mathrm{~nm}$ of $\mathrm{Nb}$ on silicon wafers by sputtering. The transition temperatures of these $\mathrm{Nb}$ films are between 6.6 to $7.2 \mathrm{~K}$. SiO film of the same thickness have been deposited on $\mathrm{Nb}$ films without any adhesion problems. We are in the process of fabricating micobolometers with a $(10 \mu \mathrm{m})^{2} \mathrm{Mo} / \mathrm{Cu}$ bilayer TES thermometer coupled to $\mathrm{Cu}$ absorbers of different geometries.

\section{Conclusions AND Future Plans}

We have outlined a plan for optimization and realization of a THM for a particularly demanding scientific application involving future measurements of the CMB. We have shown that these detectors can be designed to operate at the limit imposed by fluctuations in the arrival of the CMB photons themselves. The proposed bolometer has a total power sensitivity at $90 \mathrm{GHz}$ to a $2.7 \mathrm{~K}$ blackbody spectrum of NET $=25 \mu \mathrm{K} \sqrt{\mathrm{s}}$. Such a detector would be capable of measuring the CMB temperature anisotropy in each observed pixel in only one second of integration time. Arrays of such detectors could map out 100s of such pixels simultaneously.

\section{ACKNOWLEDGMENT}

The authors wish to acknowledge the suggestions, helpful comments, and efforts of J. Vaillancourt and L. Isenhower.

\section{REFERENCES}

[1] W. Hu and S. Dodelson, "Cosmic microwave background anisotropies," Annual Rev. of Astronomy and Astrophysics, vol. 40, pp. 171-216, 2002.

[2] K. D. Irwin, "An application of electrothermal feedback for high resolution cryogenic particle detection," Appl. Phys. Lett, vol. 60, p. 1998, 1995.

[3] J. Clarke, G. I. Hoffer, P. L. Richards, and N. H. Yeh, "Superconductive bolometers for submillimeter wavelengths," Journal of Appl. Physics, vol. 48, pp. 4865-4879, 1977.

[4] K. D. Irwin, G. C. Hilton, D. A. Wollman, and J. M. Martinis, "X-ray detection using a superconducting transition-edge sensormicrocalorimeter with electrothermal feedback," Appl. Phys. Lett., vol. 69, p. 1945, 1996.

[5] A. T. Lee, S. F. Lee, J. M. Gildemeister, and P. L. Richards, "Voltage-biased superconducting bolometers for infrared and mm-wave astronomy," in Proc. LTD-7 Munich, 1997, p. 123.

[6] J. Mees, M. Nahum, and P. L. Richards, "New designs for antennacoupled superconducting bolometers," Appl. Phys. Lett., vol. 59, pp. 2329-2331, Oct. 1991.

[7] M. Nahum, P. L. Richards, and C. A. Mears, "Design analysis of novel hot electron microbolometer," IEEE Trans. Appl. Supercond., vol. 3, pp. 2124-2127, 1993.

[8] M. Nahum and J. M. Martinis, "Ultrasensitive-hot-electron microbolometer," Appl. Phys. Lett., vol. 63, pp. 3075-3077, 1993.

[9] B. Cabrera, R. M. Clarke, P. Colling, A. J. Miller, S. Nam, and R. W. Romani, "Detection of single infrared, optical, and ultraviolet photons using superconducting transition edge sensors," Appl. Phys. Lett., vol. 73, pp. 735-737, 1998.

[10] B. S. Karasik, W. R. McGrath, M. E. Gershenson, and A. V. Sergeev, "Photon-noise-limited direct detector based on disorder-controlled electron heating," J.of Appl. Physics, vol. 87, no. 10, pp. 7586-7588, 2000.

[11] D. J. Benford, T. R. Hunter, and T. G. Phillips, "Noise equivalent power of background limited thermal detectors at submillimeter wavelengths," Int J. IR MM Waves, vol. 19, p. 931, 1998.

[12] F. C. Wellstood, C. Urbina, and J. Clarke, "Hot-electron limitation to the sensitivity of the dc superconducting quantum interference device," Appl. Phys. Lett., vol. 54, pp. 2599-2601, 1989.

[13] P. Burke, R. J. Schoelkopf, D. E. Prober, A. Skalare, B. S. Karasik, M. C. Gaidis, W. R. McGrath, B. Bumble, and H. G. LeDuc, "Mixing and noise in diffusion and phonon cooled superconducting hot electron bolometers," J. Appl. Phys., vol. 85, pp. 1644-1653, 1999.

[14] F. Pobell, Matter and Methods at Low Temperature, 2nd ed. BerlinHeidelberg: Springer, 1996

[15] G. Chattopadhyay and J. Zmuidzinas, "A dual-polarized slot antenna for millimeter waves," IEEE Trans. Antennas Propagat, vol. 46, pp. 736-737, May 1998.

[16] J. Zmuidzinas, "Quasioptical slot antenna SIS mixers," IEEE Trans. Microwave Theory Tech., vol. 40, pp. 1797-1804, Sept. 1992.

[17] M. J. Myers, A. T. Lee, P. L. Richards, D. Schwan, J. T. Skidmore, A. D. Smith, H. Spieler, and J. Yoon, "Antenna-coupled arrays of voltage-biased superconducting bolometers," in Proc. Low Temperature Detectors- Madison, 2001, pp. 247-250.

[18] A. Goldin, J. J. Bock, C. Hunt, A. E. Lange, H. LeDuc, A. Vayonakis, and J. Zmuidzinas, "SAMBA: Superconductiong antenna coupled, multifrequency,bolometric array," in Proc. Low Temperature DetectorsMadison, 2001, pp. 251-255.

[19] D. Filipovic, G. Gauthier, S. Raman, and G. Rebeiz, "Off-axis properties of silicon and quartz dielectric lens antennas," IEEE Trans. Antennas Propagat, vol. 45, pp. 760-766, May 1997.

[20] J. M. Martinis, G. C. Hilton, K. D. Irwin, and D. A. Wollman, "Calculation of $T_{c}$ in a normal superconductor bilayer using the microscopicbased Usadel theory," Nuclear Instr. Methods in Phys. Res. A, vol. 444, pp. 23-27, 2000.

[21] J. J. Bock and H. G. LeDuc, "A monolithic bolometer array suitable for FIRST," in Proc. the Far Infrared and Submillimeter Universe, 1997, p. 349.

[22] G. Chattopadhyay, D. Miller, H. G. LeDuc, and J. Zmuidzinas, "A dualpolarized quasioptical SIS mixer at $550 \mathrm{GHz}$," IEEE Trans. Microwave Theory Tech., vol. 48, pp. 1680-1686, Oct. 2000.

[23] P. Tan, L. D. Cooley, M. Galeazzi, D. Liu, D. McCammon, K. L. Nelms, and W. T. Sanders, "Mo-Cu bilayes as transition edge sensors for $\mathrm{x}$-ray astrophysics," in Proc. Low Temperature Detectors- Madison, 2001, pp. 255-258. 